\title{
Use of principal states of polarization of a liquid crystal device to achieve a dynamical modulation of broadband beams
}

\author{
Vicente Durán, ${ }^{1, *}$ Pere Clemente, ${ }^{2}$ and Jesús Lancis ${ }^{1}$ \\ ${ }^{1}$ Departament de Física, GROC-UJI, E12071 Castelló, Spain \\ ${ }^{2}$ Servei Central d'Instrumentació Científica, Universitat Jaume I, E12071 Castelló, Spain \\ *Corresponding author: vduran@sg.uji.es
}

Received May 28, 2009; revised July 8, 2009; accepted July 9, 2009;

posted July 15, 2009 (Doc. ID 111859); published August 6, 2009

\begin{abstract}
A spatially resolved polarization switcher operating over a bandwidth of $200 \mathrm{~nm}$ is demonstrated. The system is based on liquid crystal technology and no specific-purpose birefringent element is required. The procedure is founded on the polarization mode dispersion theory of optical fibers, which provides a convenient framework for the design of broadband polarization systems. Our device benefits from the high resolution of off-the-shelf twisted nematic liquid crystal displays and is well suited for spatial modulation of the intensity of broadband beams, such as those coming from few-cycle femtosecond lasers. (C) 2009 Optical Society of America

OCIS codes: $230.3720,260.5430,230.6120$.
\end{abstract}

Several proposals have been reported in recent years to use the liquid crystal (LC) technology in the implementation of broadband polarization devices, such as achromatic phase retarders or polarization switchers [1-5]. These elements are potentially striking in the applications that require a dynamical modulation of beams coming from femtosecond lasers or incoherent sources [6,7]. Much of the reported broadband devices combine several LC cells of nematic type. The wavelength dependence of such systems is minimized by optimizing the design parameters of each LC component [3-5].

In this Letter, we present an unusual approach in the field of LC devices to modulate the state of polarization (SOP) of a broadband beam. Our procedure is borrowed from the well-established polarization mode dispersion (PMD) theory in optical fibers [8]. PMD is a form of modal dispersion that has its origin in optical birefringence. This phenomenon is modeled by means of the so-called principal states of polarization (PSPs). For any concatenation of birefringent elements, the PSP model points out the existence of two input SOPs for which the corresponding output SOPs are stationary to first order in frequency [9].

Here, the PSP model is exploited to flatten the spectral response of an LC device. As a proof of concept, we have considered a system composed of a twisted nematic liquid crystal display (TNLCD) sandwiched between two liquid crystal variable retarders (LCVRs). In contrast to previous works, we have employed commercially available devices, so a control over the design parameters of LC elements is not required. Just by adjusting the configuration of the LCVRs, we have developed a switchable linear polarization rotator with a bandwidth spreading over $200 \mathrm{~nm}$. A binary intensity modulation is then attained by inserting the LC concatenation between a pair of crossed linear polarizers. A device composed of similar elements has been previously reported, but with the aim of rotating, not switching, the input polarization [2]. Furthermore, we have taken advan- tage of the pixelated structure of a TNLCD, which enables a spatial control of the input light intensity. In this way, the whole system behaves as a broadband binary intensity spatial light modulator (SLM).

Let us briefly review some results of the PMD theory useful for our purpose. We follow the notation and the theoretical discussion presented in [8]. In the absence of polarization-dependent loss, the change in polarization when light is transmitted through a birefringent medium can be described by a unitary Jones matrix $\mathbf{U}$,

$$
\mathbf{U}=\left(\begin{array}{cc}
a & b \\
-b^{*} & a^{*}
\end{array}\right)
$$

where $a$ and $b$ are complex quantities and the asterisk symbolizes the complex conjugate. The output PSPs of the medium are the eigenstates of the operator $j \mathbf{U}_{\omega} \mathbf{U}^{\dagger}$, where $j$ is the imaginary unit, the dagger stands for the Hermitian conjugate, and the subscript $\omega$ denotes differentiation at a certain angular frequency $\omega_{0},(\mathrm{~d} / \mathrm{d} \omega)_{\omega_{0}}$. The Jones vectors corresponding to the PSPs, written in the Dirac bracket notation, are determined by the eigenvalue problem

$$
j \mathbf{U}_{\omega} \mathbf{U}^{\dagger}\left|p_{ \pm}\right\rangle= \pm(\tau / 2)\left|p_{ \pm}\right\rangle .
$$

In Eq. (2), $\tau$ is the differential group delay (DGD) between the slow and fast PSPs, respectively labeled by a positive and a negative sign, respectively. Both vectors are orthogonal; i.e., their inner product is zero, $\left\langle p_{+} \mid p_{-}\right\rangle=0$.

Using the PSP model, PMD can be characterized in the three-dimensional Stokes space by means of the PMD vector $\tau$, defined as

$$
\tau=\tau \hat{p},
$$

where $\hat{p}$ is a $3 \times 1$ unit vector that points in the direction of the slow output PSP, whereas $-\hat{p}$ represents the fast output PSP. Note that Eq. (3) is written in a right-circular Stokes space. The components $\tau_{i}$ of the 
PMD vector, as well as the DGD $\tau$, are determined through the equations

$$
\begin{gathered}
\tau_{1}=2 j\left(a_{\omega} a^{*}+b_{\omega} b^{*}\right), \\
\tau_{2}=2 \operatorname{Im}\left(a_{\omega} b-b_{\omega} a\right), \\
\tau_{3}=2 \operatorname{Re}\left(a_{\omega} b-b_{\omega} a\right), \\
\tau=2 \sqrt{a_{\omega} a_{\omega}^{*}+b_{\omega} b_{\omega}^{*} .}
\end{gathered}
$$

In the above expressions $\operatorname{Im}()$ and $\operatorname{Re}($ ) denote, respectively, the imaginary and real parts of complex quantities.

The connection between an input PSP $\hat{s}$ and the corresponding output one $\hat{p}$ is governed by

$$
\hat{p}=\mathbf{M}_{R} \hat{s},
$$

where $\mathbf{M}_{R}$ is the $3 \times 3$ Mueller rotation matrix isomorphic to $\mathbf{U}$. Equation (5) describes a rotation on the surface of the Poincare sphere that transforms the input vector $\hat{s}$ into an output vector $\hat{p}$ independent to first order in frequency.

We have applied the above analysis to the LC arrangement delimited in Fig. 1 with a dotted rectangle. LCVRs are parallel-aligned LC devices without spatial structure, which provide wavelengthdependent retardances $2 \delta_{1}$ and $2 \delta_{2}$ and have their slow axes oriented at angles $\xi_{1}$ and $\xi_{2}$ from the $x$ axis, respectively. The retardance $2 \delta_{k}$ is given by $2 \delta_{k}$ $=(\omega / c) \Delta C_{k}$, where $c$ is the speed of light in vacuum and $\Delta C_{k}$ is the voltage-controlled optical path difference between the extraordinary and ordinary components of light. The polarization properties of LCVRs are described by conventional waveplate Jones matrices $\mathbf{J}_{k}(k=1,2)$. In our system, LCVRs are configured to provide the same retardance for each frequency $\left(\delta_{1}=\delta_{2}=\delta\right)$ and are oriented at normal directions $\left(\xi_{2}=\xi_{1} \pm \pi / 2\right)$. In this way, $\mathbf{J}_{1}$ and $\mathbf{J}_{2}$ are inverse matrices of each other. In its turn, the TNLCD is a pixelated device constituted by cells with a molecular twist angle $\phi$, an optical birefringence $\Delta n$, and a thickness $d$. In the off state, when no signal is addressed to the cells, their polarization properties are described by a Jones matrix $\mathbf{J}_{\mathrm{TN}}$ (off) [10],

$$
\mathbf{J}_{\mathrm{TN}}(\mathrm{off})=\exp (-j \Psi) \mathbf{R}(-\phi)\left(\begin{array}{cc}
X-j Y & Z \\
-Z & X+j Y
\end{array}\right),
$$

where $\mathbf{R}$ is a $2 \times 2$ rotation matrix, $\Psi$ is a global phase, $X=\cos \gamma, Y=(\beta / \gamma) \sin \gamma$, and $Z=(\phi / \gamma) \sin \gamma$,

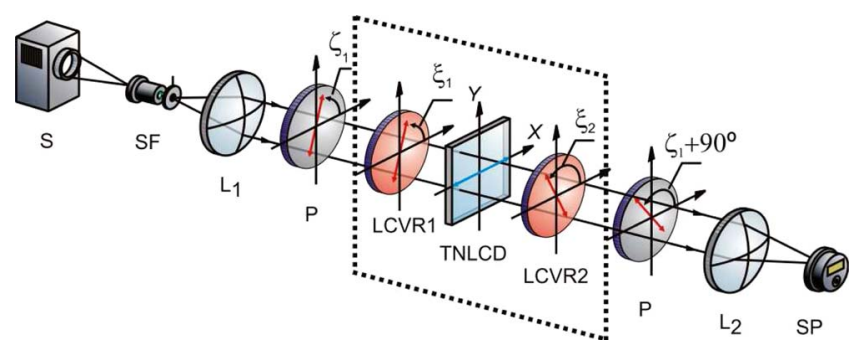

Fig. 1. (Color online) Scheme of the optical setup. The $x$ axis is parallel to the molecular director at the entrance side of the TNLCD. with $\gamma=\sqrt{\phi^{2}+\beta^{2}}$. In these expressions, $\beta$ is the birefringence angle defined as $\beta=(\omega / 2 c) \Delta n d$. In the on state, when a maximum voltage is applied to the cells, they ideally behave as a transparent isotropic media, i.e., $\mathbf{J}_{\mathrm{TN}}(\mathrm{on})=\mathbf{I}$ for all the spectral components.

The operation principle of the LC modulator is explained as follows. The Jones matrix $\mathbf{J}$ of the complete LC concatenation is given by the matrix product $\mathbf{J}=\mathbf{J}_{2} \mathbf{J}_{\mathrm{TN}} \mathbf{J}_{1}$. When a cell of the TNLCD is in the on state, the matrix $\mathbf{J}$ is simply the identity matrix, since the action of LCVRs over light polarization is mutually canceled. On contrary, when no signal is sent to a cell of the TNLCD, $\mathbf{J}$ is a unitary matrix that can be written, aside from global phase factors, as the matrix $\mathbf{U}$ of Eq. (1). By means of Eqs. (3) and (4), we can determine the components $p_{i}=p_{i}\left(\Delta C, \xi_{1}\right)$ $(i=1,2,3)$ of the slow output PSP for a given frequency $\omega_{0}$. The corresponding components $s_{i}\left(\Delta C, \xi_{1}\right)$ of the slow input PSP can be obtained though Eq. (5). If $\hat{p}$ and $\hat{s}$ are contained in the equatorial plane of the sphere, the system behaves as a switchable linear polarization rotator. Consequently, a search algorithm can be designed to find the optimal configuration of LCVRs that simultaneously minimizes the absolute value of the parameters $p_{3}\left(\Delta C, \xi_{1}\right)$ and $s_{3}\left(\Delta C, \xi_{1}\right)$.

The LC concatenation used here includes a commercial TNLCD (Sony LCX016AL with $832 \times 624$ sized pixels $32 \mu \mathrm{m}$ ) and two LCVRs (from Meadowlark Optics) calibrated for the visible spectrum. The TNLCD is composed of cells with a twist angle $\phi$ $=-1.594 \mathrm{rad}$ and $d \Delta n=0.47 \mu \mathrm{m}$ at $550 \mathrm{~nm}$. The application of a voltage to the cells is performed by sending a gray-level image to the device. Concerning the LCVRs, their slow axis can only be oriented at two orthogonal directions with respect the $x$ axis $\left(\xi_{1}\right.$ $=0,90^{\circ}$ ). The configuration of the LCVRs that minimizes the merit function $F\left(\Delta C, \xi_{1}\right)=\left(p_{3}\right)^{2}+\left(s_{3}\right)^{2}$ at $\omega_{0}$ $=3.42 \times 10^{15} \mathrm{~s}^{-1}\left(\lambda_{0}=550 \mathrm{~nm}\right)$ is $\left(\Delta C=0.4 \mu \mathrm{m}, \xi_{1}=0^{\circ}\right)$. The azimuths of the input and output PSPs are, respectively, $\zeta_{1}=-6^{\circ}$ and $\zeta_{2}=-85.5^{\circ}$, and the ellipticity angle is less than $0.2^{\circ}$ in both cases. The switchable SOP rotation leads to a binary intensity modulation by inserting the LC concatenation between two orthogonal polarizers, with the first one oriented in the direction of either the slow or the fast input PSP. As is shown in Fig. 1, the second polarizer must be ori-

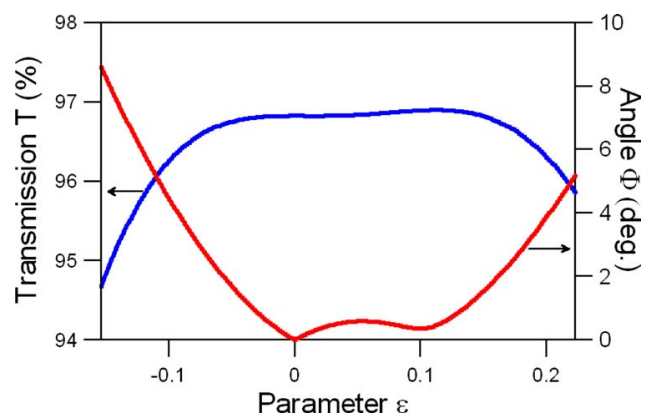

Fig. 2. (Color online) Modeled transmission in the bright state and rate of rotation of vector $\tau$ as a function of the detuning parameter $\varepsilon$, which covers the spectral range from 450 to $650 \mathrm{~nm}$. 

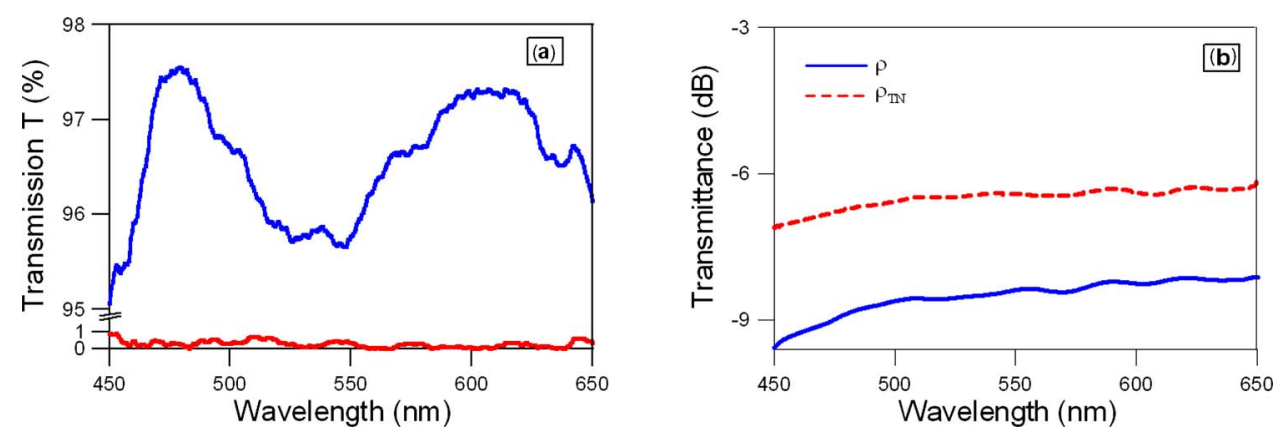

Fig. 3. (Color online) (a) Measured intensity in the bright and dark states and (b) transmittance of the SLM in the bright state and that corresponding to the sample TNLCD in the off state.

ented at $\zeta_{1}+90^{\circ}$ to ensure a minimum transmission when the TNLCD is in the on state. In this way, SLM presents two operation modes (dark and bright) depending on the signal addressed to the TNLCD. Figure 2 shows the transmittance $T$ for the bright state versus the detuning parameter $\varepsilon$, defined as $\varepsilon$ $=\Delta \omega / \omega_{0}$ with $\Delta \omega=\omega-\omega_{0}$. A mean transmission of $96.5 \%$ is achieved with a residual intensity variation $\Delta T$ lower than $2.2 \%$. Such an intensity response is explained by the small wavelength dependence of the SOP impinging onto the analyzer, which has an azimuth that varies $2^{\circ}$ from 450 to $650 \mathrm{~nm}$, with a mean ellipticity angle of $0.3^{\circ}$. These results clearly improve those obtained from a single TNLCD, which produces an intensity variation of the order of $10 \%$ when it is inserted between crossed polarizers [2]. Furthermore, our system has the advantage of not requiring the fabrication of specific LC structures, although specially designed devices present a wider achromatic response [3-5].

The PMD vector is stationary only to the first order, so a residual chromatism still remains. Secondorder PMD is defined as the frequency derivative of $\boldsymbol{\tau}$, $\tau_{\omega}=\mathrm{d} \tau / \mathrm{d} \omega$. In accordance with Eq. (3), $\boldsymbol{\tau}_{\omega}$ can be written as the sum of two perpendicular vectors, $\tau_{\omega}=\tau_{\omega} \hat{p}$ $+\tau \hat{p}_{\omega}$. Here we are interested only in the angular rate of rotation of the vector $\tau,\left|\hat{p}_{\omega}\right|=\mathrm{d} \Phi / \mathrm{d} \omega$, where $\Phi$ is the angle between $\boldsymbol{\tau}\left(\omega_{0}+\Delta \omega\right)$ and $\boldsymbol{\tau}\left(\omega_{0}\right)$ [8]. In Fig. 2 the variation in $\Phi$ with the parameter $\varepsilon$ is depicted for our system. A comparison with $T(\varepsilon)$ shows that for $-0.03<\varepsilon<0.14$ (from 485 to $570 \mathrm{~nm}$ ) $\Phi<1^{\circ}$ and $T$ is practically constant. Out of this range, $\left|\hat{p}_{\omega}\right|$ takes a nonnegligible value, so $\Phi$ continually increases leading to a more significant intensity variation.

The above results have been experimentally verified with the optical setup shown in Fig. 1. We used as a white light source (S) a xenon arc lamp followed by a spatial filter $(\mathrm{SF})$ and a collimating lens $\left(\mathrm{L}_{1}\right)$. The LC concatenation was sandwiched between two linear polarizers (P). Spectral intensity was measured with the aid of a focusing lens $\left(\mathrm{L}_{2}\right)$ and a spectrophotometer (SP). Experimental data were normalized to unit by measuring the total light intensity impinging onto the detector. Figure 3(a) shows the SLM transmission $T$ in the dark state (mean extinction ratio of $3 \times 10^{-3}$ ) and in the bright state (mean transmission of about $96.6 \%$ with $\Delta T<2.5 \%$ ). To take into account losses caused by internal reflections and by the TNLCD pixelated structure, we show in Fig. 3(b) the transmittance $\rho$ of the SLM in the bright state, defined as $\rho=10 \log \left(I_{1} / I_{0}\right)$, where $I_{0}$ and $I_{1}$ are, respectively, the light intensity after the first and second polarizers. The slope of $\rho$ in the short wavelength range is a consequence of the light absorption by the TNLCD. The transmittance of the display (without polarizers) in the off state, $\rho_{\mathrm{TN}}$, is also included in Fig. 3(b). The mean distance between both curves is 2 $\mathrm{dB}$, with a standard deviation of $0.15 \mathrm{~dB}$.

In summary, we have demonstrated that the PSP model, usually limited to characterizing polarization effects in optical fibers, constitutes an efficient tool to flatten the spectral response of a LC device. As a proof of concept, we have implemented a binary intensity SLM that includes only commercially available elements. Experimental results show a flat bright response over a spectral band of $200 \mathrm{~nm}$ in the visible region (root mean square error lower than 6 $\times 10^{-3}$ ) with an extinction ratio of about 1:1000 using high-quality polarizers.

This research was funded by the Spanish Ministry of Education and Science (under projects FIS200762217 and Consolider Program SAUUL CSD200700013).

\section{References}

1. P. Hariharan and P. E. Ciddor, Opt. Eng. 36, 952 (1997).

2. Z. Zhuang, Y. J. Kim, and J. S. Patel, Appl. Phys. Lett. 76, 3995 (2000).

3. M. D. Lavrentovich, T. A. Sergan, and J. R. Kelly, Opt. Lett. 29, 1411 (2004).

4. S. Shen, J. She, and T. Tao, J. Opt. Soc. Am. A 22, 961 (2005).

5. Q. Wang, G. Farrell, T. Freir, and J. She, J. Opt. A 7, 47 (2005).

6. M. Bock, S. K. Das, R. Grunwald, S. Osten, P. S. Staudt, and G. Stibenz, Appl. Phys. Lett. 92, 151105 (2008).

7. L. Martínez-León, P. Clemente, E. Tajahuerce, G. Mínguez-Vega, O. Mendoza-Yero, M. FernándezAlonso, J. Lancis, V. Climent, and P. Andrés, Appl. Phys. Lett. 94, 011104 (2009).

8. N. Damask, Polarization Optics in Telecommunications (Springer, 2005).

9. C. D. Poole and R. E. Wagner, Electron. Lett. 22, 1029 (1986).

10. K. H. Lu and B. E. A. Saleh, Opt. Eng. 29, 240 (1990). 